\title{
THE LOCAL GREEN'S FUNCTION METHOD IN SINGULARLY PERTURBED CONVECTION-DIFFUSION PROBLEMS
}

\author{
OWE AXELSSON, EVGENY GLUSHKOV, AND NATALYA GLUSHKOVA
}

\begin{abstract}
Previous theoretical and computational investigations have shown high efficiency of the local Green's function method for the numerical solution of singularly perturbed problems with sharp boundary layers. However, in several space variables those functions, used as projectors in the Petrov-Galerkin scheme, cannot be derived in a closed analytical form. This is an obstacle for the application of the method when applied to multi-dimensional problems. The present work proposes a semi-analytical approach to calculate the local Green's function, which opens a way to effective practical application of the method. Besides very accurate approximation, the matrix stencils obtained with these functions allow the use of fast and stable iterative solutions of the large sparse algebraic systems that arise from the grid-discretization. The advantages of the method are illustrated by numerical examples.
\end{abstract}

\section{INTRODUCTION}

Singularly perturbed problems are generally acknowledged to be a hard task for numerical evaluation. Due to their solutions having sharp boundary and interior layers severe numerical instability can occur and a large error pollution spreads out over the whole domain as the perturbation parameter tends to its limit value.

A classical example of a singularly perturbed equation is the equation of the convection-diffusion problem:

$$
\mathcal{L} u \equiv-\varepsilon \Delta u+\mathbf{b} \cdot \nabla u+c u=f, \quad(x, y) \in \Omega \subset R^{2}
$$

where $\varepsilon$ is a small parameter and $c \geq 0$. Here we denote scalar products of algebraic vectors by dots, as in the convection term $\mathbf{b} \cdot \nabla u$, while the brackets notation is reserved for the scalar products in the functional Hilbert spaces occurring below.

For the sake of definiteness let us consider a rectangular domain $\Omega: 0 \leq x \leq$ $a_{1}, 0 \leq y \leq a_{2}$ with homogeneous Dirichlet boundary condition

$$
\left.u\right|_{\Gamma}=0, \quad \Gamma=\partial \Omega .
$$

Only the presence of the diffusion term $-\varepsilon \Delta u$ enables fulfillment of this condition at the outflow part $\Gamma_{-}$of the boundary $\Gamma$ entailing a boundary layer of width $O(\varepsilon)$ near $\Gamma_{-}$(here $\Gamma_{-}=\{(x, y) \in \Gamma: \mathbf{b} \cdot \mathbf{n}>0\}, \mathbf{n}$ is an outward normal to $\partial \Omega$ ).

It is well known that if the standard Galerkin method, or the similar central difference method, is used in regions where layers occur, then unphysical oscillations

Received by the editor March 25, 2003 and, in revised form, January 9, 2007.

2000 Mathematics Subject Classification. Primary 65F10, 65N22, 65R10, 65R20.

Key words and phrases. Convection-diffusion equation, Petrov-Galerkin discretization, Fourier transform, integral equations, iterative solution. 


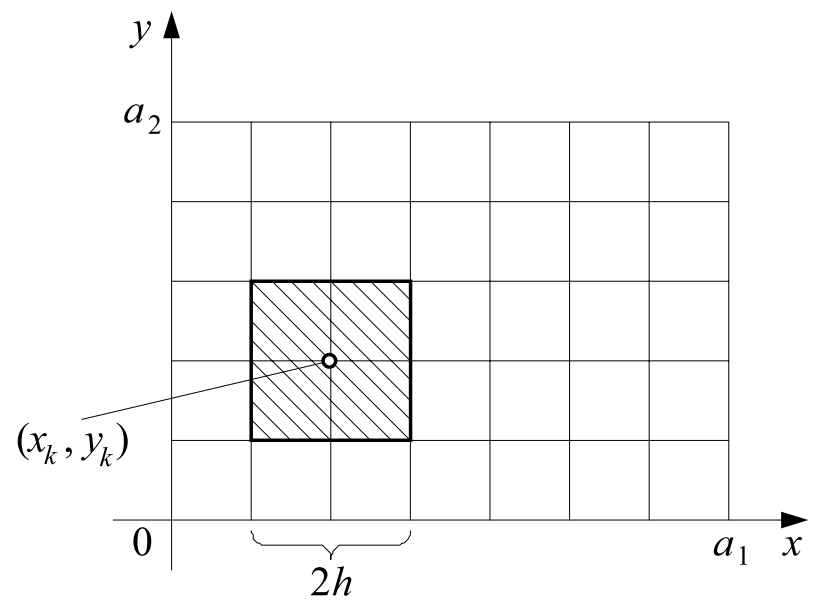

FiguRe 1

arise. They can be suppressed by the use of a locally and significantly refined grid where a certain local Peclét number condition is satisfied [1]. Instead of the standard Galerkin method, the streamline upwind method (see 2]) is a popular method to stabilize the scheme.

Other methods used are related to the local characteristic line method, which is based on the property that away from the layers the solution follows narrowly the characteristic lines for the reduced equation $(\varepsilon=0)$, when $\varepsilon$ is small. Using such methods with a proper locally refined grid, under certain assumptions regarding the influence of corner singularities, one can prove optimal order discretization error estimates, typically of $O\left(h^{2}\right)$, which hold uniformly in the singular perturbation parameter; see 3 , 4. Other related papers can be found in 5 .

The present paper deals with the local Green's function method. We give its main idea with a simple example of a uniform grid approximation. Let

$$
u_{h}(x, y)=\sum_{k=1}^{N} u_{k} \varphi_{k}(x, y)
$$

be an expansion of the exact solution of the problem (1.1) and (1.2) in terms of basis functions $\varphi_{k}=\varphi\left(\left(x-x_{k}\right) / h,\left(y-y_{k}\right) / h\right)$ defined at the interior nodes $\left(x_{k}, y_{k}\right)$ of a grid covering $\Omega$ with a step $h$ (Figure 1 ); $\varphi(x, y)$ is a shape-function. In line with the Petrov-Galerkin scheme, the unknown coefficients $u_{k}$ are determined from the variational condition

$$
\left(\mathcal{L} u_{h}-f, \psi_{l}\right)=0, \quad l=1,2, \ldots, N,
$$

where the set of projectors $\left\{\psi_{l}\right\}_{l=1}^{N}$ is dense as $N \rightarrow \infty$ in the Hilbert space with the scalar product of (1.4). Here we take the $L_{2}$ space with the product $(f, g)=$ $\iint f g^{*} d x d y$ (an asterisk, hereinafter, will indicate complex conjugate values).

Using the adjoint operator $\hat{\mathcal{L}}:(\mathcal{L} u, v)=(u, \hat{\mathcal{L}} v)$ the variational equality (1.4) can be converted into the form

$$
\left(u_{h}-u, \hat{\mathcal{L}} \psi_{l}\right)=0 .
$$


For equation (1.1) the operator $\hat{\mathcal{L}}$ has the form

$$
\hat{\mathcal{L}} u \equiv-\varepsilon \Delta u-\nabla \cdot(\mathbf{b} u)+c u .
$$

If the shape-function $\psi$ of the projectors $\psi_{l}=\psi\left(x-x_{l}, y-y_{l}\right)$ complies with the equality

$$
\hat{\mathcal{L}} \psi=\delta, \quad(x, y) \in \omega
$$

where $\delta(x, y)$ is Dirac's delta-function, then it follows from (1.5) that the approximate and the exact solutions coincide at the nodes

$$
u_{h}\left(x_{l}, y_{l}\right)=u\left(x_{l}, y_{l}\right) \text {. }
$$

In (1.7) $\omega$ is a finite support of $\psi$. For a uniform grid projectors $\psi_{l}$ are localized in $2 h \times 2 h$ squares centered at the nodes (Figure 1), i.e. in such a case $\omega=\omega_{h}$ is a square $|x| \leq h,|y| \leq h$. In accordance with the general scheme, $\psi$ must satisfy the natural boundary condition

$$
\left.\psi\right|_{\Gamma_{h}}=0,
$$

where $\Gamma_{h}$ is the boundary of $\omega_{h}$. The shape-function $\psi(x, y)$ is the local Green's function of the problem considered [1].

In practice the local Green's function method exhibits high accuracy at the nodes even with a coarse grid. It is further of significant importance that appearance of the layers as $\varepsilon \rightarrow 0$ does not degrade its numerical stability. Besides the theoretical heuristic calculations above, this fact was shown numerically as well, however, only with 1D examples 6, 1]. Extension of the method to several variables has been restrained by the absence of an analytical solution of problem (1.7), (1.9) for such a case. The present work gives an example of an effective computer implementation of the method in 2D by using a semi-analytical Fourier-transform technique.

It is pertinent to note that the local Green's function can be given not only in a square. Since its main property is to give a delta-function in (1.5), we can vary the shape of $\omega_{h}$ regardless of supports of trial functions. It is only important to set the test local Green's functions at points, where coincidence (1.8) should be achieved.

We start from the square support $\omega_{h}$, which goes together with a uniform grid discretization, just to demonstrate that the method works and works well without any mesh refinement in the layer parts of the domain. If more points within a layer are desirable, one can take, for example, rectangular supports with arbitrary large aspect ratio. This allows one to adjust the mesh in the layers along straight lines to become arbitrarily thin along the layer. The generalization of the technique given below on such a case is obvious. It would only require using two mesh values $h_{1}, h_{2}$ instead of $h$ in (2.8) $-(2.15)$ and further on.

As the most promising with a non-uniform (non-polygonal) mesh we mention the use of circle and elliptic supports. The solution of integral equations at $\partial \omega$ and derivation of integral's asymptotics as $\varepsilon \rightarrow 0$ (see Section 2) can be much easier for such subdomains with smooth boundaries than for polygonal elements with corner points.

In this paper we give, first, an idea of the technique proposed with a 1D example (subsection 2.1), extending then its application to the 2D case (subsection 2.2). The local Green's function $\psi(x, y)$ is derived in terms of integrals with respect to the unknown normal derivative $\partial \psi / \partial n$ at $\Gamma_{h}$. The latter, in turn, is approximated by orthogonal polynomials. Then, in Section 3, we derive the computational formulae 
for the Petrov-Galerkin method arriving at a matrix stencil also expressed in terms of $\partial \psi / \partial n$. Some numerical test examples are given in Section 4. Finally, stability bounds and error estimates are discussed in Section 5.

\section{Integral RePRESENTATion of the LOCAL GREen's FunCtions}

For an efficient derivation of the local Green's functions we use the Fourier transform technique. The Fourier transform pair

$$
\begin{gathered}
\left\{\begin{array}{l}
\mathcal{F}[u] \equiv \int_{R^{n}} u(\mathbf{x}) e^{i \boldsymbol{\alpha} \cdot \mathbf{x}} d \mathbf{x}=U(\boldsymbol{\alpha}), \\
\mathcal{F}^{-1}[U] \equiv \frac{1}{(2 \pi)^{n}} \int_{R^{n}} U(\boldsymbol{\alpha}) e^{-i \boldsymbol{\alpha} \cdot \mathbf{x}} d \boldsymbol{\alpha}=u(\mathbf{x}),
\end{array}\right. \\
\mathbf{x}=\left(x_{1}, x_{2}, \ldots, x_{n}\right), \boldsymbol{\alpha}=\left(\alpha_{1}, \alpha_{2}, \ldots, \alpha_{n}\right), \boldsymbol{\alpha} \cdot \mathbf{x}=\sum_{i}^{n} \alpha_{i} x_{i}
\end{gathered}
$$

can only be applied to differential equations with constant coefficients. However, since the discretization step $h$ is assumed to be much smaller than the characteristic scales of the variation of the coefficients, we can approximate $\mathbf{b}(\mathbf{x})$ and $c(\mathbf{x})$ in (1.1) by piecewise functions, which are constant within element subdomains (the so-called freezing coefficients concept). In this case we take $\nabla \cdot(\mathbf{b} \psi)=\mathbf{b} \cdot \nabla \psi$ with $\mathbf{b}=$ const in (1.7). By virtue of the variational statement of the problem, below we treat operator $\mathcal{F}$ as a generalized Fourier transform applicable to distributions (Dirac's $\delta$-functions) as well.

2.1. 1D case. To get an idea of the method proposed, let us first apply the transform to the 1D two-point problem (1.7), (1.9), whose solution is actually easily derived by other means as well. Application of the transform presumes that a function is defined in the whole space $R^{n}$, so we extend $\psi(x)$ to the exterior of the interval $|x| \leq h$ by zero. In this case the points $x= \pm h$ are the points of discontinuity for derivatives $\psi^{(m)}(x), m \geq 1(\psi(x)$ is continuous due to the boundary condition (1.9): $\psi( \pm h)=0$ ). Fourier transform of derivatives of non-smooth functions obeys the following rule:

$$
\mathcal{F}\left[u^{(m)}\right]=(-i \alpha)^{m} U(\alpha)+\left(u_{m-1}-i \alpha u_{m-2}+\cdots+(-i \alpha)^{m-1} u_{0}\right) e^{i \alpha x_{0}},
$$

where $U(\alpha)=\mathcal{F}[u], x_{0}$ is a point of discontinuity and $u_{m}=\lim _{\delta \rightarrow 0}\left[u^{(m)}\left(x_{0}-\delta\right)\right.$ $\left.-u^{(m)}\left(x_{0}+\delta\right)\right]$ are values of the jumps of the derivatives at $x_{0}$. Therefore, the Fourier transform brings (1.7), extended by zero onto the whole axis, to the functional equation

$$
\mathcal{F}[\hat{\mathcal{L}} \psi]=L(\alpha) \Psi(\alpha)-v^{+} e^{i \alpha h}+v^{-} e^{-i \alpha h}=1 .
$$

Here, $L(\alpha)=\varepsilon \alpha^{2}+i \alpha b+c ; v^{ \pm}=\varepsilon \psi^{\prime}( \pm h \mp 0)$ are unknown constants, which have to be taken to meet the boundary conditions $\psi( \pm h)=0$.

From (2.2) we get immediately

$$
\Psi(\alpha)=G(\alpha)\left(1+v^{+} e^{i \alpha h}-v^{-} e^{-i \alpha h}\right), \quad G(\alpha)=1 / L(\alpha),
$$

and then

$$
\psi(x)=\mathcal{F}^{-1}[\Psi(\alpha)]=g(x)+v^{+} g(x-h)-v^{-} g(x+h),
$$


where

$$
g(x)=\mathcal{F}^{-1}[G]=\frac{1}{2 \pi} \int_{-\infty}^{\infty} G(\alpha) e^{-i \alpha x} d \alpha
$$

is the global Green's function of (1.7) in 1D.

An explicit representation of $g(x)$ is easily derived from the integral (2.4) by means of the residual technique for path integrals. There are two pure imaginary poles of $G(\alpha)$ (roots of the denominator $L(\alpha)=\varepsilon\left(\alpha-\zeta_{1}\right)\left(\alpha-\zeta_{2}\right)$ ) located in the upper and lower half-planes of the complex plane $\alpha$ :

$$
\zeta_{1,2}=i\left(-b \pm \sqrt{b^{2}+4 \varepsilon c}\right) / 2 \varepsilon .
$$

In accordance with Jordan's lemma and Cauchy's theorem [7, the contribution of the residuals at poles $\zeta_{1}$ for $x \leq 0$ and $\zeta_{2}$ for $x \geq 0$ yields

$$
\begin{aligned}
g(x) & =\frac{i}{\varepsilon\left(\zeta_{1}-\zeta_{2}\right)}\left\{\begin{array}{cl}
e^{-i \zeta_{1} x}, & x \leq 0 \\
e^{-i \zeta_{2} x}, & x \geq 0
\end{array}\right\} \\
& \sim \frac{1}{b}\left\{\begin{array}{cl}
e^{c x / b}, & x \leq 0 \\
e^{-\left(b^{2}+c \varepsilon\right) x / \varepsilon b}, & x \geq 0
\end{array}\right\}, \quad \text { as } \quad \varepsilon \rightarrow 0
\end{aligned}
$$

For small $\varepsilon$, the function $g(x)$ demonstrates a typical layer behaviour at the right (upwind) side of the source-point $x=0$ and an independent of $\varepsilon$ decrease for $x<0$ (the dash-point line in the example shown in Figure 2). Consequently, in (2.3) the term $g(x+h)$ has a layer at $x=-h$ while $g(x-h)$ does not contribute to any sharp layer of $\psi(x)$ (dashed lines). After fixing the values $v^{ \pm}$from the condition $\psi( \pm h)=0$, the total plot of $\psi(x)$ takes the form shown by the solid line in Figure 2.

It may seem attractive to design $\psi(\mathbf{x})$ in $2 \mathrm{D}$ using $1 \mathrm{D}$ functions of the form (2.3) taken, for example, along the characteristics of $\hat{\mathcal{L}}$. However, it cannot be performed analytically. Moreover, since in several variables $\mathbf{x}=0$ is a singular point (with a logarithmic singularity in 2D: $g(\mathbf{x}) \sim g_{0} \ln |\mathbf{x}|$, as $|\mathbf{x}| \rightarrow 0$ ), any approximation by such functions would be qualitatively mismatched. Special methods for $\psi(\mathbf{x})$ designed in $R^{n}, n \geq 2$ must therefore be developed.

2.2. 2D case. Similarly to the above, application of the Fourier transform to (1.7) in the entire space $R^{2}$ yields the explicit integral representation for the global Green's function $g(x, y)$ :

$$
\begin{gathered}
g(\mathbf{x})=\frac{1}{(2 \pi)^{2}} \int_{-\infty}^{\infty} G(\boldsymbol{\alpha}) e^{-i \boldsymbol{\alpha} \cdot \mathbf{x}} d \boldsymbol{\alpha}, \quad G(\boldsymbol{\alpha})=1 / L(\boldsymbol{\alpha}) \\
L(\boldsymbol{\alpha})=\varepsilon|\boldsymbol{\alpha}|^{2}+i \boldsymbol{\alpha} \cdot \mathbf{b}+c
\end{gathered}
$$

However, it can be simplified to one-fold integrals only:

$$
\begin{aligned}
& g(x, y)=\frac{1}{2 \pi} \int_{-\infty}^{\infty}\left\{\begin{array}{cc}
G_{1}\left(\alpha_{2}, x\right) e^{-i \alpha_{2} y} d \alpha_{2}, & |x| \geq|y| \\
G_{2}\left(\alpha_{1}, y\right) e^{-i \alpha_{1} x} d \alpha_{1}, & |y| \geq|x|
\end{array}\right\}, \\
& G_{n}\left(\alpha, x_{n}\right)=\exp \left[-\left(b_{n} x_{n}+d_{n}\left|x_{n}\right|\right) / 2 \varepsilon\right] / d_{n}, \quad n=1,2, \\
& d_{n}(\alpha)=\sqrt{b_{n}^{2}+4 \varepsilon\left(\varepsilon \alpha^{2}+i \hat{b}_{n} \alpha+c\right)}, \quad \hat{b}_{n}= \begin{cases}b_{2}, & n=1, \\
b_{1}, & n=2,\end{cases}
\end{aligned}
$$




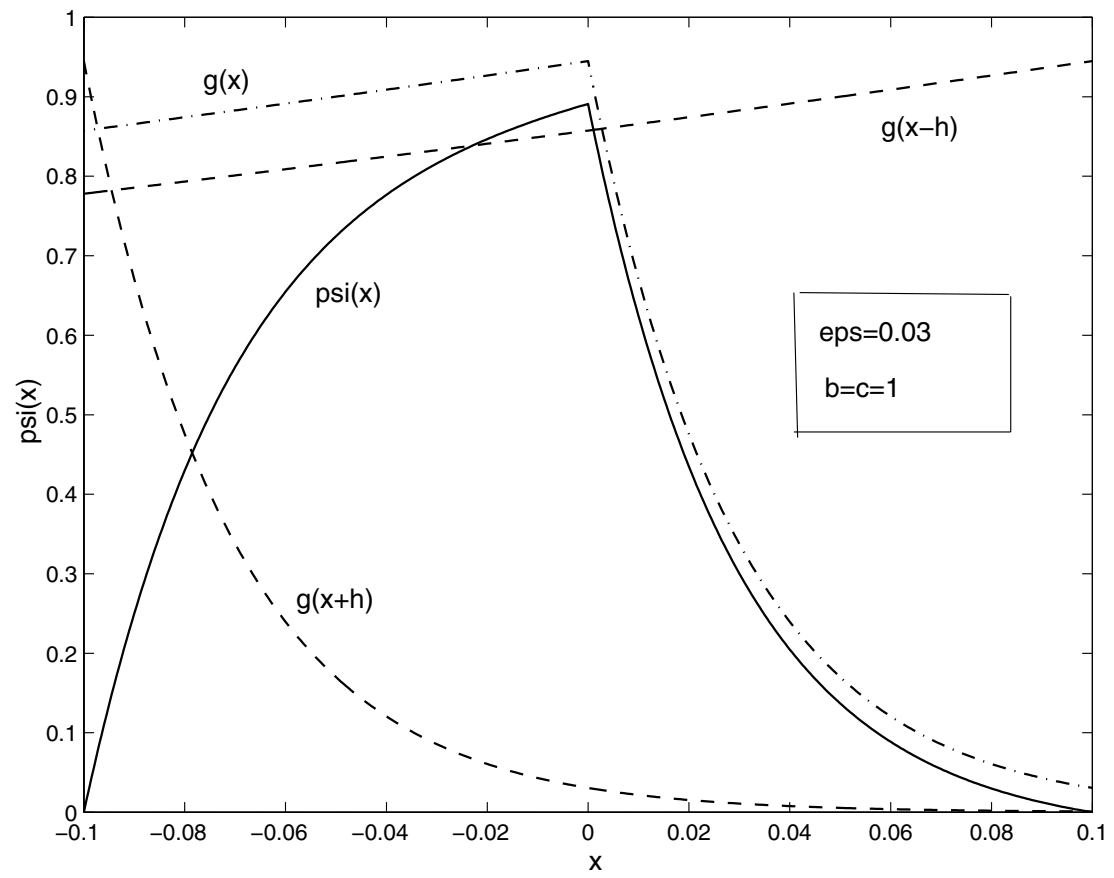

Figure 2. 1D local Green's function

(whenever no confusion can arise, we write $\alpha$ instead of $\alpha_{1}$ or $\alpha_{2}$ for the components of $\boldsymbol{\alpha}$ and $x, y$ instead of $x_{1}, x_{2}$ for the $\mathbf{x}$ ones). Further simplification of these integrals in line with the residual technique is impossible due to the branch points of $d_{n}(\alpha)$ and the branch cuts implied in the complex plane $\alpha$.

When $r=\sqrt{x^{2}+y^{2}} \rightarrow 0$, as expected, these integrals become divergent in accordance with the logarithmic singularity of $g(x, y)$. We remark that selection of the integrals in (2.7) depending on the $|x|,|y|$ ratio is not mandatory. It only provides its faster convergence at infinity $($ as $\alpha \rightarrow \infty)$.

If $\psi$ is considered to be extended by zero from $\omega_{h}$ onto the whole plane $R^{2}$, then (1.7) takes in the Fourier transform domain in the following form:

$$
\begin{array}{r}
\Psi(\boldsymbol{\alpha})=G(\boldsymbol{\alpha})\left\{1+V_{x}^{+}\left(\alpha_{2}\right) e^{i \alpha_{1} h}-V_{x}^{-}\left(\alpha_{2}\right) e^{-i \alpha_{1} h}\right. \\
\left.+V_{y}^{+}\left(\alpha_{1}\right) e^{i \alpha_{2} h}-V_{y}^{-}\left(\alpha_{1}\right) e^{-i \alpha_{2} h}\right\} .
\end{array}
$$

Here $V_{x}^{ \pm}=\mathcal{F}\left[v_{x}^{ \pm}\right]$and $V_{y}^{ \pm}=\mathcal{F}\left[v_{y}^{ \pm}\right]$, where $v_{x}^{ \pm}(y)$ and $v_{y}^{ \pm}(x)$ are unknown normal derivatives $\varepsilon \partial \psi / \partial x$ and $\varepsilon \partial \psi / \partial y$ at the square sides $x= \pm h$ and $y= \pm h$, respectively. Fourier inversion of (2.8) implies

$$
\psi=g+u_{x}^{+}-u_{x}^{-}+u_{y}^{+}-u_{y}^{-}
$$


with functions

(2.10)

$$
\left\{\begin{array}{l}
u_{x}^{ \pm}(x, y)=\frac{1}{2 \pi} \int_{-\infty}^{\infty} G_{1}(\alpha, x \mp h) V_{x}^{ \pm}(\alpha) e^{-i \alpha y} d \alpha=\int_{-h}^{h} g(x \mp h, y-t) v_{x}^{ \pm}(t) d t \\
u_{y}^{ \pm}(x, y)=\frac{1}{2 \pi} \int_{-\infty}^{\infty} G_{2}(\alpha, y \mp h) V_{y}^{ \pm}(\alpha) e^{-i \alpha x} d \alpha=\int_{-h}^{h} g(x-t, y \mp h) v_{y}^{ \pm}(t) d t
\end{array}\right.
$$

expressed in terms of the unknown $v_{x}^{ \pm}$and $v_{y}^{ \pm}$.

In view of boundary condition (1.9), these unknowns have to comply with the integral equations

$$
\begin{cases}\left.\left(u_{x}^{+}-u_{x}^{-}+u_{y}^{+}-u_{y}^{-}\right)\right|_{x= \pm h}=-g( \pm h, y), & |y| \leq h, \\ \left.\left(u_{x}^{+}-u_{x}^{-}+u_{y}^{+}-u_{y}^{-}\right)\right|_{y= \pm h}=-g(x, \pm h), & |x| \leq h .\end{cases}
$$

A semi-analytical solution of these equations can be obtained in terms of certain basis functions $p_{k}(t),|t| \leq h$ :

$$
\begin{cases}v_{x}^{ \pm}(y)=\sum_{k=0}^{\infty} c_{k}^{ \pm} p_{k}(y), & |y| \leq h \\ v_{y}^{ \pm}(x)=\sum_{k=0}^{\infty} t_{k}^{ \pm} p_{k}(x), & |x| \leq h\end{cases}
$$

if Galerkin's scheme of discretisation is applied to (2.11).

Since the unknowns are expected to be continuous functions, the usual Fourier exponents $e^{ \pm i \pi k t / h}$ or splines are quite acceptable as a basis. However, orthogonal polynomials with a weight accounting for the behaviour at the ends of the approximation interval generally provide a better convergence. For the numerical examples below we have selected $p_{k}(t)=\left(h^{2}-t^{2}\right) P_{k}^{(1,1)}(t / h)$ as the basis and $q_{l}(t)=P_{l}(t / h)$ as test functions $\left(P_{k}^{(\alpha, \beta)}(x), P_{l}(x)\right.$ are Jacobi and Legendre polynomials 8$\left.]\right)$.

Galerkin's variational procedure reduces (2.11) to the linear algebraic system

$$
\sum_{k=0}^{M} A_{l k} \mathbf{t}_{k}=\mathbf{f}_{l}, \quad l=0,1,2, \ldots, M
$$

for the unknown expansion coefficients $\mathbf{t}_{k}=\left\{c_{k}^{+}, c_{k}^{-}, t_{k}^{+}, t_{k}^{-}\right\} ; M$ fixes the number of terms kept in expansion (2.12). With the basis and projectors selected, the $4 \times 4$ matrix-blocks $A_{l k}$ and the right-hand side vectors $\mathbf{f}_{l}$ take the form

$$
A_{l k}=\left[\begin{array}{cccc}
a_{1, l k}(0) & -a_{1, l k}(2 h) & b_{2, l k}^{+}(h) & -b_{2, l k}^{-}(h) \\
a_{1, l k}(-2 h) & -a_{1, l k}(0) & b_{2, l k}^{+}(-h) & -b_{2, l k}^{-}(-h) \\
b_{1, l k}^{+}(h) & -b_{1, l k}^{-}(h) & a_{2, l k}(0) & -a_{2, l k}(2 h) \\
b_{1, l k}^{+}(-h) & -b_{1, l k}^{-}(-h) & a_{2, l k}(-2 h) & -a_{2, l k}(0)
\end{array}\right]
$$




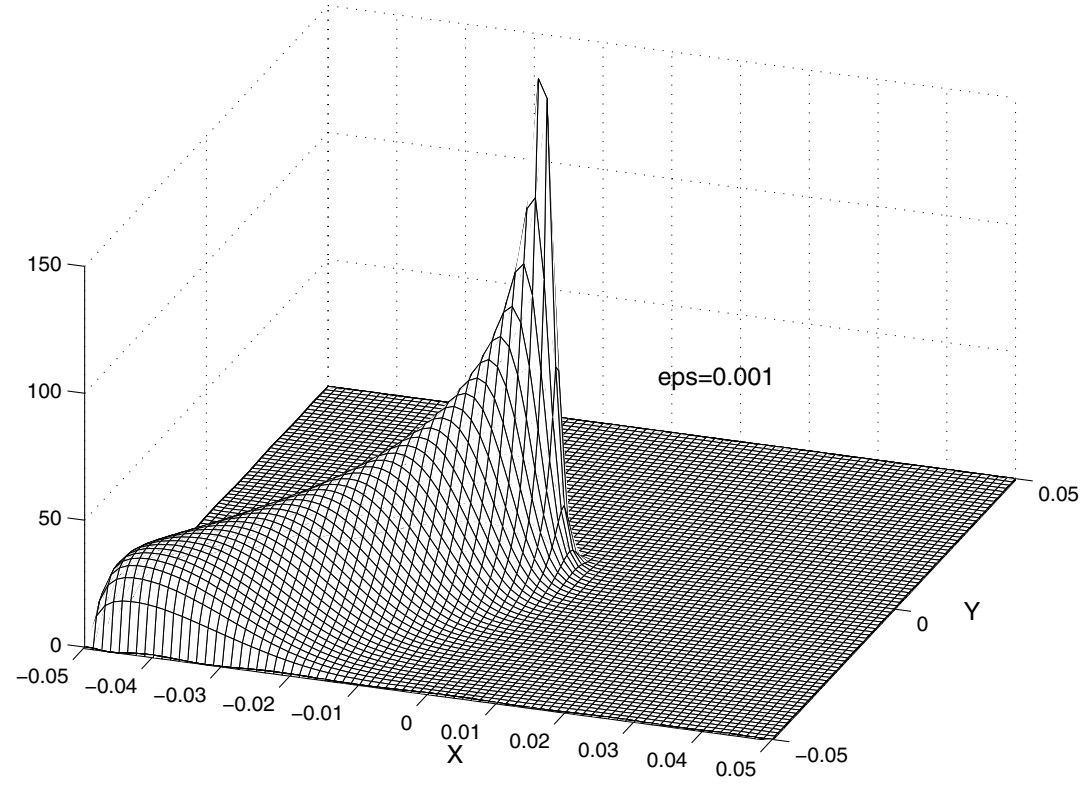

Figure 3. 2D local Green's function

where

$$
\begin{aligned}
& \left\{\begin{array}{l}
a_{n, l k}(2 p)=\frac{1}{2 \pi} \int_{-\infty}^{\infty} G_{n}(\alpha, 2 p) P_{k}(\alpha) Q_{l}(\alpha) d \alpha, \\
b_{n, l k}^{ \pm}(p)=\frac{1}{2 \pi} \int_{-\infty}^{\infty} e_{n}^{ \pm}(\alpha) P_{k}(\alpha) Q_{l}\left(z_{n}^{ \pm}\right) e^{-i \alpha p} d \alpha, \\
f_{n, l}^{ \pm}=-\frac{1}{2 \pi} \int_{-\infty}^{\infty} G_{n}(\alpha, \pm h) Q_{l}(\alpha) d \alpha,
\end{array}\right. \\
& p=0, \pm h ; \quad n=1,2 ; \quad z_{n}^{ \pm}=i\left( \pm d_{n}-b_{n}\right) / 2 \varepsilon, \\
& e_{n}^{ \pm}(\alpha)=\exp \left(\left(-d_{n} \pm b_{n}\right) \lambda / 2\right) / d_{n}, \lambda=h / \varepsilon, \\
& G_{n}\left(\alpha, x_{n}\right), d_{n}(\alpha) \text { as in (2.7), } \\
& P_{k}(\alpha)=\mathcal{F}\left[p_{k}\right]=i^{k} 4(k+1) h^{2} j_{k+1}(\alpha h) / \alpha, \\
& Q_{l}(\alpha)=\mathcal{F}\left[q_{l}(\alpha)\right]^{*}=i^{-l} 2 h j_{l}(\alpha h),
\end{aligned}
$$

$j_{m}(z)=\sqrt{2 \pi / z} J_{m+1 / 2}(z)$ are spherical Bessel functions [8].

Despite the fact that integrals (2.15) are improper ones given over an infinite interval, due to factors such as $e^{-d_{n} \lambda}$, their convergence is very fast, although certain a priori analytical calculation aiming to adjust them to the numerical integration has to be carried out. Moreover, if $\varepsilon \ll 1$ and, consequently, $\lambda=h / \varepsilon$ is very large, the exponential behavior of the integrands permits very good approximation of the integrals by saddle-point asymptotics, derived using the steepest descent method [9].

An example of the shape function $\psi(x, y)$ computed in line with the scheme above for $\varepsilon=0.001, h=0.05, c=1$ and a diagonal velocity field $\mathbf{b}=\{\sqrt{2}, \sqrt{2}\} / 2$ is given in Figure 3. Similar to the 1D example above one can see a sharp decrease on the upwind side of the source-point $\mathbf{x}=0$ and boundary layers at the outflow part 
of the edges $x=-0.05$ and $y=-0.05$ (for the backward flux $-\mathbf{b}$ associated with the adjoint operator $\hat{\mathcal{L}}$ ). The growth in accordance with the logarithmic singularity at $\mathbf{x}=0$, which does not appear in the $1 \mathrm{D}$ case, is also clearly seen here.

However, it is important to note the fact that we do not need $\psi(x, y)$ itself, but due to the integral technique used, the matrix stencil we seek can be obtained merely in terms of the auxiliary functions $v_{x}^{ \pm}$and $v_{y}^{ \pm}$.

\section{The MATRIX-STENCIL}

The variational equality (1.4) results in an algebraic system

$$
\sum_{k=1}^{N} a_{l k} u_{k}=f_{l}, \quad l=1,2, \ldots, N,
$$

where $a_{l k}=\left(\mathcal{L} \varphi_{k}, \psi_{l}\right), f_{l}=\left(f, \psi_{l}\right)$, and $u_{k}$ are unknown coefficients in expansion (1.3). The generalized Parseval equality $(f, g)=\frac{1}{(2 \pi)^{2}}(F, G)$ makes it possible to evaluate the components $a_{l k}$ in the Fourier transform domain:

$$
a_{l k}=\frac{1}{(2 \pi)^{2}}\left(\mathcal{F}\left[\mathcal{L} \varphi_{k}\right], \Psi_{l}\right) .
$$

Here, for $\mathcal{F}\left[\mathcal{L} \varphi_{k}\right]=L(\boldsymbol{\alpha}) \Phi_{k}(\boldsymbol{\alpha})$ and $\Psi_{l}(\boldsymbol{\alpha})=\mathcal{F}\left[\psi_{l}\right]=\Psi(\boldsymbol{\alpha}) e^{i\left(\alpha_{1} x_{l}+\alpha_{2} y_{l}\right)}$, all the functions are assumed to be extended by zero beyond their supports.

Let the basis shape-function $\varphi$ be designed from the traditional hat functions

$$
\varphi(x, y)=\varphi(x) \varphi(y), \quad \varphi(t)=\left\{\begin{array}{cc}
1-|t|, & |t| \leq 1 \\
0, & |t|>1
\end{array}\right.
$$

so that $\Phi_{k}(\boldsymbol{\alpha})=\mathcal{F}\left[\varphi_{k}\right]=h^{2} \Phi\left(\alpha_{1} h\right) \Phi\left(\alpha_{2} h\right) e^{i\left(\alpha_{1} x_{k}+\alpha_{2} y_{k}\right)}$ with $\Phi(\alpha)=\mathcal{F}[\varphi]$.

Since the function $G(\boldsymbol{\alpha})$, involved in the representation (3.2) through $\Psi(\boldsymbol{\alpha})$ of form (2.8), is the inverse to $L(\boldsymbol{\alpha}): L(\boldsymbol{\alpha}) G(\boldsymbol{\alpha})=1$, this representation can be simplified to the form

$$
a_{l k}=\delta(l-k)+a_{l k, x}^{+}-a_{l k, x}^{-}+a_{l k, y}^{+}-a_{l k, y}^{-},
$$

where $\delta(p)=\left\{\begin{array}{l}1, p=0, \\ 0, p \neq 0\end{array}\right.$ is the Kronecker delta,

$$
\begin{gathered}
\left\{\begin{array}{l}
a_{l k, x}^{ \pm}=\delta\left(p_{1} \pm 1\right) I_{p_{2}}^{ \pm}, \\
a_{l k, y}^{ \pm}=\delta\left(p_{2} \pm 1\right) J_{p_{1}}^{ \pm},
\end{array}\right. \\
\left\{\begin{array}{l}
I_{p_{2}}^{ \pm}=\int_{-h}^{h} v_{x}^{ \pm}(y) \varphi\left(y / h+p_{2}\right) d y, \\
J_{p_{1}}^{ \pm}=\int_{-h}^{h} v_{y}^{ \pm}(x) \varphi\left(x / h+p_{1}\right) d x,
\end{array}\right. \\
I_{p_{2}}^{ \pm}, J_{p 1}^{ \pm} \equiv 0 \text { for }\left|p_{n}\right| \geq 2, n=1,2, \\
p_{1}=\left(x_{l}-x_{k}\right) / h, p_{2}=\left(y_{l}-y_{k}\right) / h, p_{n}=0, \pm 1 .
\end{gathered}
$$


The restriction $\left|p_{n}\right| \leq 1$ means that only nodes $\left(x_{k}, y_{k}\right)$ adjacent to $\left(x_{l}, y_{l}\right)$ contribute to $a_{l k}$. The structure of the three by three matrix-stencil $B=\left[b\left(p_{1}, p_{2}\right)\right]$, fixing components $a_{l k}$ in line with the rule $a_{l k}=b\left(p_{1}, p_{2}\right), p_{n}=-1,0,1$, is therefore easily seen:

$$
B=\left[\begin{array}{ccc}
I_{-1}^{+}+J_{-1}^{+} & I_{0}^{+} & I_{1}^{+}-J_{-1}^{-} \\
J_{0}^{+} & 1 & -J_{0}^{-} \\
-I_{-1}^{-}+J_{1}^{+} & -I_{0}^{-} & -I_{1}^{-}-J_{1}^{-}
\end{array}\right] .
$$

As soon as the expansion coefficients $\mathbf{t}_{k}$ have been determined from the system (2.13), calculation of the entries of the $B$-matrix through integrals (3.6) does not take any major part in the total computing time.

Besides that, representation (3.7) is helpful for analyzing general properties of the sparse-matrix $A=\left[a_{l k}\right]$ with regards to the iterative solution of system (3.1). Since $\partial \psi / \partial n<0$ at $\Gamma_{h}$ (due to a decrease of $\psi$ from positive values to zero when approaching the edges) all entries of $B$, except $b(0,0)=1$, are negative. It results in negative off-diagonal components of $A$, so that $A$ is an $M$-matrix if it is diagonally dominant [10] (see also subsection 5.1).

\section{NumericAl EXAMPLES}

4.1. Boundary layers. As an example let us consider problems (1.1)-(1.2) in the unit square $0 \leq x, y \leq 1$ with the right-hand side function $f(x, y)$ generated by the exact solution

$$
\begin{gathered}
u(x, y)=x^{2} y^{2}\left(1-e_{1}(x)\right)\left(1-e_{2}(y)\right), \\
e_{n}\left(x_{n}\right)=\exp \left(-\left(1-x_{n}\right) / \varepsilon\right), \quad n=1,2 \quad x_{1}=x, x_{2}=y,
\end{gathered}
$$

with boundary layers at the sides $x=1$ and $y=1$.

In actual computation $f(x, y)$ was replaced by a piecewise function, so that the components $f_{l}$ in system (3.1) were approximated as follows:

$$
f_{l}=\left(f, \psi_{l}\right) \approx f\left(x_{l}, y_{l}\right) \iint \psi_{l} d \Omega=f\left(x_{l}, y_{l}\right)\left[1+4\left(c_{0}^{+}-c_{0}^{-}+t_{0}^{+}-t_{0}^{-}\right) / 3\right] / c
$$

(if $c=0$ the method is also applicable but with another form of the right-hand side $\left.f_{l}\right)$.

The coefficients in (1.1) are assumed to be constant; $c=1$ in all the cases. The velocity vector $\mathbf{b}$ has unit length $|\mathbf{b}|=1$, its direction is defined by the angle $\theta: \mathbf{b}=(\cos \theta, \sin \theta)$. In this case the Peclét number is equal to $h /(2 \varepsilon)$.

The examples given in Tables 1 and 2 show how the matrix-stencil $B$ and the solution get stabilized as $\varepsilon \rightarrow 0$, despite the sharp boundary layers occurring in the exact solution (4.1). 
Table 1. $\theta=0$

\begin{tabular}{|c|c|c|c|c|c|c|}
\hline$\varepsilon$ & \multicolumn{3}{|c|}{ matrix-stencil $B$} & number of & error $r_{h}$ & time \\
\hline $10^{-1}$ & $\begin{array}{l}-0.0843 \\
-0.1622 \\
-0.0909\end{array}$ & $\begin{array}{c}-0.1543 \\
1.0000 \\
-0.1705\end{array}$ & $\begin{array}{l}-0.0843 \\
-0.1622 \\
-0.0909\end{array}$ & 990 & 0.0057 & 14 sec. \\
\hline $10^{-2}$ & $\begin{array}{l}-0.0563 \\
-0.1528 \\
-0.1196\end{array}$ & $\begin{array}{c}-0.0914 \\
1.0000 \\
-0.2485\end{array}$ & $\begin{array}{l}-0.0563 \\
-0.1528 \\
-0.1196\end{array}$ & 301 & 0.0046 & $5 \mathrm{sec}$. \\
\hline $10^{-3}$ & $\begin{array}{l}-0.0002 \\
-0.0128 \\
-0.1596\end{array}$ & $\begin{array}{c}-0.0000 \\
1.0000 \\
-0.2485\end{array}$ & $\begin{array}{l}-0.0002 \\
-0.0128 \\
-0.1596\end{array}$ & 40 & 0.0058 & $2 \mathrm{sec}$. \\
\hline $10^{-5}$ & $\begin{array}{l}-0.0000 \\
-0.0000 \\
-0.0292\end{array}$ & $\begin{array}{c}-0.0000 \\
1.0000 \\
-0.9315\end{array}$ & $\begin{array}{l}-0.0000 \\
-0.0000 \\
-0.0292\end{array}$ & 17 & 0.0030 & $6 \mathrm{sec}$. \\
\hline $10^{-7}$ & $\begin{array}{l}-0.0000 \\
-0.0000 \\
-0.0255\end{array}$ & $\begin{array}{c}-0.0000 \\
1.0000 \\
-0.9389\end{array}$ & $\begin{array}{l}-0.0000 \\
-0.0000 \\
-0.0255\end{array}$ & 16 & 0.0028 & 29 sec. \\
\hline $10^{-9}$ & $\begin{array}{l}-0.0000 \\
-0.0000 \\
-0.0255\end{array}$ & $\begin{array}{c}-0.0000 \\
1.0000 \\
-0.9390\end{array}$ & $\begin{array}{l}-0.0000 \\
-0.0000 \\
-0.0255\end{array}$ & 16 & 0.0028 & $113 \mathrm{sec}$. \\
\hline
\end{tabular}

TABle 2. $\theta=\pi / 8$

\begin{tabular}{|c|c|c|c|c|c|c|}
\hline$\varepsilon$ & \multicolumn{3}{|c|}{ matrix-stencil $B$} & number of & error $r_{h}$ & time \\
\hline $10^{-1}$ & $\begin{array}{l}-0.083 \\
-0.159 \\
-0.089\end{array}$ & $\begin{array}{c}-0.1549 \\
1.0000 \\
-0.1699\end{array}$ & $\begin{array}{l}-0.0858 \\
-0.1653 \\
-0.0920\end{array}$ & 4950 & 0.0113 & 66 sec. \\
\hline $10^{-2}$ & $\begin{array}{l}-0.049 \\
-0.125 \\
-0.101\end{array}$ & $\begin{array}{c}-0.0952 \\
1.0000 \\
-0.2397\end{array}$ & $\begin{array}{l}-0.0673 \\
-0.1846 \\
-0.1336\end{array}$ & 282 & 0.0039 & 5 sec. \\
\hline $10^{-3}$ & $\begin{array}{l}-0.000 \\
-0.001 \\
-0.045\end{array}$ & $\begin{array}{l}-0.0001 \\
1.0000 \\
-0.5184\end{array}$ & $\begin{array}{l}-0.0012 \\
-0.0743 \\
-0.3490\end{array}$ & 21 & 0.0021 & $2 \mathrm{sec}$. \\
\hline $10^{-5}$ & $\begin{array}{l}-0.000 \\
-0.0000 \\
-0.003\end{array}$ & $\begin{array}{c}-0.0000 \\
1.0000 \\
-0.5717\end{array}$ & $\begin{array}{l}-0.0000 \\
-0.0000 \\
-0.4136\end{array}$ & 9 & 0.0030 & $7 \mathrm{sec}$. \\
\hline $10^{-7}$ & $\begin{array}{l}-0.000 \\
-0.000 \\
-0.005\end{array}$ & $\begin{array}{l}-0.0000 \\
1.0000 \\
-0.5695\end{array}$ & $\begin{array}{l}-0.0000 \\
-0.0000 \\
-0.4147\end{array}$ & 9 & 0.0025 & 43 sec. \\
\hline $10^{-9}$ & $\begin{array}{l}-0.000 \\
-0.000 \\
-0.005\end{array}$ & $\begin{array}{c}-0.0000 \\
1.0000 \\
-0.5695\end{array}$ & $\begin{array}{l}-0.0000 \\
-0.0000 \\
-0.4147\end{array}$ & 9 & 0.0025 & 116 sec. \\
\hline
\end{tabular}


In these tables elements of the matrix-stencil $B$, the number of Seidel iterations for system (3.1), the average nodal error and the computing time are given depending on the singular parameter $\varepsilon$. We keep only four digits in the elements of $B$, so that zero ones are in reality small but non-zero. The number of iterations was determined by the relative accuracy $10^{-11}$. The error $r_{h}=\int\left|u-u_{h}\right| d \Omega$ is approximated by the nodal sum $h^{2} \sum_{k}\left|u\left(x_{k}, y_{k}\right)-u_{h}\left(x_{k}, y_{k}\right)\right|, h^{2}=1 / N$. The number of grid cells in these examples was $N=100 \times 100$, that is, $h=0.01$. The number of expansion terms $M$ (see 2.12, 2.13) in all examples was taken to be equal to 10 . Table 1 is for the velocity parallel to the $x$-axis, $\theta=0$, while Table 2 is for $\theta=\pi / 8$. The computations were carried out on a PC with the processor speed $733 \mathrm{MHz}$. The increase of the total computing time for very small values of $\varepsilon$ is due to the cost of the numerical calculation of the integrals (2.15). As was mentioned above, these computing expenses are practically reduced to zero if those integrals are replaced by their asymptotic expressions for large Peclét numbers.

The next table demonstrates how convergence, accuracy and computing time depend on the grid size $h$.

TABLE 3. $\varepsilon=0.0001, \theta=\pi / 8$

\begin{tabular}{|c|c|c|c|c|}
\hline grid $N_{1} \times N_{2}=N$ & grid size $h$ & $\begin{array}{c}\text { number of } \\
\text { iterations }\end{array}$ & error $r_{h}$ & total time \\
\hline $100 \times 100=10^{4}$ & 0.01 & 6 & 0.00353 & 3 sec. \\
\hline $200 \times 200=4 \cdot 10^{4}$ & 0.005 & 9 & 0.00172 & 3 sec. \\
\hline $300 \times 300=9 \cdot 10^{4}$ & 0.0033 & 11 & 0.00100 & 8 sec. \\
\hline $400 \times 400=16 \cdot 10^{4}$ & 0.0025 & 20 & 0.00066 & 14 sec. \\
\hline $500 \times 500=25 \cdot 10^{4}$ & 0.0020 & 27 & 0.00036 & $25 \mathrm{sec}$. \\
\hline
\end{tabular}

4.2. Interior layers. The examples above show that the method behaves stably despite the presence of boundary layers. However, since in general there were no mesh points in the layers, it can make a wrong impression that the algorithm is only applicable in regions where the reduced first order problem approximates the exact solution. To demonstrate that it has the same favorable accuracy properties in other regions we include the next test example with interior layers (Figure 4). Here the equation (1.1) is homogeneous $(f \equiv 0)$ but with an inhomogeneous boundary condition at the inflow part $\Gamma_{+}$instead of (1.2): $\left.u\right|_{\Gamma}=u_{0}$ where $u_{0}=1$ for $x=0$, $0.25 \leq y \leq 0.5$ and $u_{0} \equiv 0$ for the rest of $\Gamma$.

To take into account this inhomogeneous condition, we add a known function $u_{0, h}:\left.u_{0, h}\right|_{\Gamma}=u_{0}$ to $u_{h}$ of form (1.3). By doing so, the matrix $\left[a_{l k}\right]$ of the system (3.1) remains the same, while $u_{0, h}$ only changes the right-hand side into $f_{l}=$ $-\left(\mathcal{L} u_{0, h}, \psi_{l}\right)$.

As was expected, the numerical results show two interior layers along the characteristic lines emanating from the points of discontinuity of $u_{0}(0,0.25)$ and $(0,0.5)$ at $\Gamma_{+}$and a downstream boundary layer at the outflow side $x=1$. As is seen, no unphysical oscillations occur and the method is able to model the behavior of the exact solution accurately with no need to use any additional points in the layer domain. 


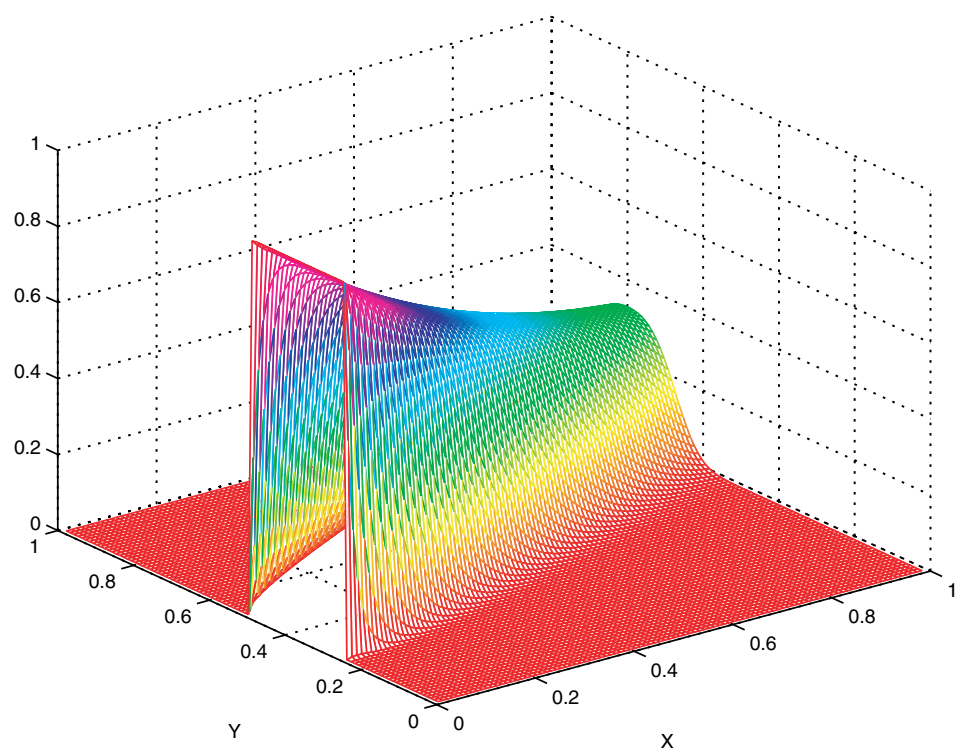

Figure 4. Test example with interior layers emanating from the points of discontinuity of the boundary condition at the inflow side; $\varepsilon=10^{-7}, \theta=\pi / 8, h=0.01$.

\section{AnAlysis of the nUmerical PROCEDUREs}

In this section we survey various techniques relevant to the present study, to prove stability and derive discretization error estimates. We also comment briefly on the use of a hybrid method, i.e. a combination of the standard Galerkin and Petrov-Galerkin methods. As we shall see, it is the Petrov-Galerkin part, with proper test functions, which provides the stabilization of the methods.

5.1. Stability bounds. We recall first stability bounds for approximations of the problem (1.1)-(1.2). The first is associated with its variational formulation

$$
a(u, v)=\int_{\Omega} f v d \Omega, \quad \text { for all } v \in \stackrel{\circ}{H}^{1}(\Omega)
$$

where

$$
a(u, v)=\int_{\Omega}(\varepsilon \nabla u \cdot \nabla v+\mathbf{b} \cdot \nabla u v+c u v) d \Omega,
$$

and the norm

$$
\|u\|_{1, \varepsilon}:=\left[\varepsilon|u|_{1}^{2}+\|u\|^{2}\right]^{\frac{1}{2}} .
$$

For these, coercivity

$$
\rho\|u\|_{1, \varepsilon}^{2} \leq a(u, u) \quad \text { for all } u \in \stackrel{\circ}{H}^{1}(\Omega)
$$

can be readily proven to hold with $\rho=\min \left\{1, c_{0}\right\}$ (see e.g. [1]) if

$$
\min _{\Omega}\left(c-\frac{1}{2} \nabla \cdot \mathbf{b}\right) \geq c_{0}>0 .
$$


We shall assume that (5.2) holds and in addition, $c \geq 0$ in $\Omega$. As shown in [1, even if the given velocity field $\mathbf{b}$ does not satisfy (5.2), it can hold for a properly transformed differential equation. Together with the boundedness estimate

$$
a(u, u) \leq\|f\|\|u\|,
$$

it shows that there exists a unique solution, which satisfies $\|u\|_{1, \varepsilon} \leq \frac{1}{\rho}\|f\|$.

The other useful stability estimate follows from a local Green's function $\psi_{i}$, defined on the support $\omega_{i}$ of the corresponding local basis function $\varphi_{i}$, associated with the node $\mathbf{x}_{i}$ :

$$
\begin{aligned}
& \hat{\mathcal{L}} \psi_{i}=\delta\left(\mathbf{x}-\mathbf{x}_{i}\right), \quad \mathbf{x} \in \omega_{i}, \\
& \left.\psi_{i}\right|_{\partial \omega_{i}}=0 .
\end{aligned}
$$

Assume that $\psi_{i}$ is obtained explicitly from (5.3) in terms of normal derivatives $\left.\varepsilon \frac{\partial \psi_{i}}{\partial n}\right|_{\partial \omega_{i}}$ in accordance with the scheme described in Subsection 2.2. Then

$$
\begin{aligned}
& \int_{\omega_{i}} f \psi_{i} d \Omega=\int_{\omega_{i}} \mathcal{L} u \psi_{i} d \Omega \\
& \quad=\int_{\omega_{i}}\left(\varepsilon \nabla u \cdot \nabla \psi_{i}-\nabla \cdot\left(\mathbf{b} \psi_{i}\right) u+c u \psi_{i}\right) d \Omega+\oint_{\partial \omega_{i}}\left(\varepsilon \frac{\partial u}{\partial n}+\mathbf{b} \cdot \mathbf{n} u\right) \psi_{i}=\hat{a}\left(u, \psi_{i}\right),
\end{aligned}
$$

where

$$
\hat{a}(u, v)=\int_{\omega_{i}}(\varepsilon \nabla u \cdot \nabla v-\nabla \cdot(\mathbf{b} v) u+c u v) d \Omega .
$$

Hence

$$
\int_{\omega_{i}} f \psi_{i} d \Omega=\int_{\omega_{i}}\left(-\varepsilon \Delta \psi_{i}-\nabla \cdot\left(\mathbf{b} \psi_{i}\right)+c \psi_{i}\right) u d \Omega+\oint_{\partial \omega_{i}} \varepsilon \frac{\partial \psi_{i}}{\partial n} u,
$$

or by (5.3),

$$
u\left(\mathbf{x}_{i}\right)+\oint_{\partial \omega_{i}} \varepsilon \frac{\partial \psi_{i}}{\partial n} u=\int_{\omega_{i}} f \psi_{i} d \Omega, \quad i=1, \ldots, N .
$$

Now let $u$ be approximated by bilinear finite element functions and let $u_{h}$ be the corresponding approximation also satisfying (5.4). For simplicity, we assume that the integrals in (5.4) with $u$ replaced by $u_{h}$ can be computed exactly. It can be written in a matrix form

$$
G_{h} \mathbf{U}_{h}=\mathbf{F}_{h}
$$

where $G_{h}$ is the corresponding finite element matrix and $\mathbf{U}_{h}, \mathbf{F}_{h}$ are the vectors corresponding to the nodal values of $u_{h}$ and $\int_{\omega_{i}} f \psi_{i}$, respectively. When $u_{h}$ is of form (1.3), (3.3) and $\omega_{i}$ are $2 h \times 2 h$ squares, we arrive naturally to the same system (3.1) with matrix $G_{h}=A=\left[a_{l k}\right]$ fixed by stencil (3.7).

Since $\partial \psi_{i} / \partial n \leq 0$ on $\partial \omega_{i}$ and

$$
G_{h} \mathbf{e} \geq 0 \text { for } \mathbf{e}=\left[\begin{array}{llll}
1 & 1 & \ldots & 1
\end{array}\right]^{T},
$$

it follows that $G_{h}$ is a diagonally dominant $M$-matrix. In particular, the elements of $G_{h}^{-1}$ are non-negative. We now make some additional, sufficient assumptions to guarantee that $\left\|G_{h}^{-1}\right\|_{\infty}$ is bounded by a constant $C$, independent of the number of nodepoints and the aspect ratio of the elements. If such a bound holds, it follows from (5.5) that

$$
\max _{\mathbf{x}_{i} \in \Omega_{h}}\left|u\left(\mathbf{x}_{i}\right)\right|=\left\|\mathbf{U}_{h}\right\|_{\infty} \leq C\left\|\mathbf{F}_{h}\right\|_{\infty} .
$$


The additional assumption made would guarantee that there exists a so-called barrier function $w$ (which clearly must be non-negative), such that $G_{h} \mathbf{W}_{h} \geq \mathbf{e}$. The following are examples of some sufficient conditions for the existence of such a barrier function.

Assume then that $b_{1} \geq b_{0}>0$ in $\Omega$, where $b_{0}$ is a constant. Then take $w=x$, for which

$$
\left(G_{h} \mathbf{W}_{h}\right)\left(\mathbf{x}_{i}\right)=\left(b_{1} w_{x}+c w\right)\left(\mathbf{x}_{i}\right) \geq b_{1} \geq b_{0} .
$$

Hence $\left\|G_{h}^{-1}\right\| \leq \frac{1}{b_{0}}\left\|\mathbf{W}_{h}\right\|_{\infty}=\frac{1}{b_{0}}$.

Another sufficient condition is that $\mathbf{b}$ is a potential vector field, that is, there exists a positive scalar function $\varphi$ such that $\mathbf{b}=\nabla \varphi$. In addition, assume that $|\mathbf{b}|^{2} \geq b_{0}>0$ and $\nabla \cdot \mathbf{b} \leq 0$ in $\Omega$. Then

$$
\mathcal{L} \varphi \geq|\mathbf{b}|^{2} \geq b_{0} \text { in } \Omega \text {. }
$$

Note that $-\nabla \cdot \mathbf{b}=-\Delta \varphi$, so $\varphi \geq 0$ if $\left.\varphi\right|_{\partial \Omega} \geq 0$. Therefore, $\varphi$ can be used as a barrier function.

We also make the additional assumption that $\mathbf{b}$ is a smooth vector field so that $\varphi$ is a smooth function, which can be arbitrarily closely approximated for sufficiently small values of $h$ by finite element approximations $\varphi_{h}$, where

$$
a\left(\varphi_{h}, v_{h}\right)=a\left(\varphi, v_{h}\right) \quad \text { for all } v_{h} \in V_{h} .
$$

This discretization satisfies then (approximately) the same bound as in (5.5) and, in this case,

$$
\left\|G_{h}^{-1}\right\| \leq \frac{\left\|\varphi_{h}\right\|_{\infty}}{b_{0}} \leq \frac{\|\varphi\|_{\infty}}{b_{0}}
$$

where, due to the maximum principle, $\varphi$ is bounded.

5.2. Error estimates and a hybrid approach. To provide a proper background for the error estimation, we recall first the standard Galerkin method, which takes the form

$$
a\left(u, v_{h}\right)=\int_{\Omega} f v_{h} d \Omega
$$

or

$$
a\left(u-u_{h}, v_{h}\right)=0, \quad \text { for all } v_{h} \in V_{h} \subset \stackrel{\circ}{H}^{1}(\Omega),
$$

where $V_{h}$ is a finite element space. To estimate the discretization error $e_{h}=u-u_{h}$, we split it as $e_{h}=\eta-\theta_{h}$, where $\eta=u-u_{I_{h}}, u_{I_{h}}$ is the interpolant to $u$ on $V_{h}$ and $\theta_{h}=u_{h}-u_{I_{h}}$. Then $a\left(\theta_{h}, \theta_{h}\right)=a\left(\eta, \theta_{h}\right)$ or, using coercivity,

$$
\rho\left\|\theta_{h}\right\|_{1, \varepsilon}^{2} \leq C\left[\varepsilon|\eta|_{1}^{2}+\min \left\{\varepsilon^{-1 / 2}|\eta|,|\eta|_{1}\right\}^{2}+\|\eta\|^{2}\right] .
$$

This shows that

$$
\left\|\theta_{h}\right\|_{1, \varepsilon}<C \min \left\{\varepsilon^{-1 / 2}\|\eta\|_{1, \varepsilon^{2}},\|\eta\|_{1}\right\}
$$

and, by the triangle inequality,

$$
\left\|e_{h}\right\|_{1, \varepsilon} \leq C \min \left\{\varepsilon^{-1 / 2}\|\eta\|_{1, \varepsilon^{2}},\|\eta\|_{1}\right\} .
$$

If $u \in H^{1+\nu}(\Omega), 0<\nu \leq 1$ and if $V_{h}$ is spanned by piecewise linear basis functions, we then find

$$
\left\|u-u_{h}\right\|_{1, \varepsilon} \leq C \min \left\{\left(\varepsilon^{1 / 2}+\varepsilon^{-1 / 2} h\right) h^{\nu}, h^{\nu}\right\}\|u\|_{1+\nu} .
$$

In practice, however, due to boundary layers, the derivatives of the solution grow typically as $O\left(\varepsilon^{-1 / 2-\nu}\right)$ as $\varepsilon \rightarrow 0$ and, unless $h \leq O(\varepsilon)$, this $\varepsilon$-dependence 
causes unphysical wiggles in the numerical solution, which spread into the whole domain. Therefore, one must use some cut-off weight function, which suppresses the influence of the layer part. Exponential weight functions were used in [1] and more general weight functions in [2] and [1]. It allows one to derive stable solutions and estimates in the layer free domain of $O(h), h \rightarrow 0$ which holds uniformly in $\varepsilon$.

An alternative way to handle these oscillations is to use a Petrov-Galerkin method of the following form. Find $u_{h} \in V_{h}$ such that

$$
\hat{a}\left(u_{h}, v_{h}^{\prime}\right)=\hat{a}\left(u, v_{h}^{\prime}\right), \quad \text { for all } v_{h}^{\prime} \in V_{h}^{\prime} \subset \stackrel{\circ}{H^{1}}(\Omega),
$$

where $V_{h}$ is spanned by standard basis functions but $V_{h}^{\prime}$ in general by other basis functions. In particular, we are here interested in the case where $V_{h}^{\prime}$ contains local Green's functions.

As we have seen, due to the upwind shape of the local Green's functions, they work as cut-off functions. In particular, it suffices to use them in the layer domain, i.e., the Petrov-Galerkin method is used only there while the standard Galerkin method is used in $\Omega_{\text {int }}$, the combination of both methods constitutes a hybrid approach, which stabilizes the method.

An alternative heuristic explanation of this effect can be given using the classical Aubin-Nitsche duality argument. Given the solution $u_{h}$ of the (hybrid) PetrovGalerkin method in (5.7), we let $\varphi$ be the solution of the adjoint equation,

$$
\hat{\mathcal{L}} \varphi=u-u_{h} \text { in } \Omega, \quad \frac{\partial \varphi}{\partial n}=0 \text { on } \partial \Omega
$$

(assuming here for simplicity Dirichlet boundary conditions in (5.1)). Then

$$
\int_{\Omega}\left(u-u_{h}\right)^{2} d \Omega=\int_{\Omega} \hat{\mathcal{L}} \varphi\left(u-u_{h}\right) d \Omega
$$

and a calculation shows that

$\left\|u-u_{h}\right\|^{2}=\int_{\Omega}\left[\varepsilon \nabla\left(u-u_{h}\right) \cdot \nabla\left(\varphi-v_{h}^{\prime}\right)-\nabla \cdot \mathbf{b}\left(\varphi-v_{h}^{\prime}\right)\left(u-u_{h}\right)+c\left(u-u_{h}\right)\left(\varphi-v_{h}^{\prime}\right)\right] d \Omega$.

We split the integral in two parts, $\int_{\Omega}=\int_{\Omega_{0}}+\int_{\Omega_{1}}$, where $\Omega_{1}$ contains the boundary layer and $\Omega_{0}=\Omega_{\text {int }}$. The direction of the flow for the adjoint equation is opposite to that of the given equation. Therefore, the function $\varphi$ may have a layer at the inflow boundary but not at the outflow for the primal problem. This means that, locally, the factors $u-u_{h}$ and $\nabla \cdot\left(\mathbf{b}\left(\varphi-v_{h}^{\prime}\right)\right)$ and $\nabla\left(u-u_{h}\right)$ and $\nabla\left(\varphi-v_{h}^{\prime}\right)$ can be expected to balance each other in $\Omega_{0}$ and $\Omega_{1}$, respectively, so when one is big the other is correspondingly small. If $V_{h}^{\prime}$ contains fundamental solutions at an outflow layer, the corresponding local error $\varphi-v_{h}^{\prime}$ is $\varepsilon$-independent and may even be zero. Hence, in this way, the boundary layers do not influence the global error.

\section{Conclusion}

For a 2D convection-diffusion problem we have demonstrated high practical efficiency of the local Green's function method (LGFM) for the numerical solution of singularly perturbed problems when a semi-analytic technique of LGFs construction is developed. We propose to use a Fourier transform technique, which yields the LGFs in terms of 1D contour integrals with respect to the global Green's function and unknown normal derivatives at the boundary of LGF's supports. Analytical 
approximation of the latter in terms of orthogonal polynomials is derived from the integral equations in line with the Galerkin scheme.

The grid-discretization with the LGFs leads to sparse algebraic systems with $M$-matrices permitting the use of classical iterative solution methods. It becomes clear that the smaller the singular parameter is, the faster the convergence of the iterative solution is. In other words, the worse a singularly perturbed problem is, the more effective is the LGFM application for the iterative solution. In doing so, if an asymptotic calculation of the arising integrals is done, then the cost of obtaining the matrix-stencil becomes practically negligible.

Another way to reduce the cost spent for the construction of the LGFs for each mesh node with a variable coefficients problem is to use a hybrid method, where the standard Galerkin scheme is used in the major part of the domain, while the Petrov-Galerkin LGFM discretization is confined to subdomains where boundary or interior layers occur. The LGFM suppresses the well-known unphysical oscillations, which are inherent in the Galerkin method when it runs against the layer. Such a hybrid scheme, suggested in 1 for 1D problems, has to work in a multidimensional case as well.

For 3D problems the general scheme of the method remains the same. The local Green's function and the matrix-stencil are expressed in terms of unknown normal derivative on the mesh element surface, which is obtained from the boundary integral equations.

\section{ACKNOWLEDGEMENTS}

We are thankful to Dr. B. Polman and Dr. S. Gololobov for their fruitful comments and useful remarks.

The work was supported by the NWO-RFBR 047-008-007, CRDF REC-004 and RFBR r2003yug grants.

\section{REFERENCES}

[1] O. Axelsson, Stability and error estimates of Galerkin finite element approximations for convection-diffusion equations, IMA J. Numer. Anal., 1(1981), pp. 329-345. MR641313 (83a:65105)

[2] U. Nävert, A finite element method for convection-diffusion problems, Ph.D. thesis, Chalmers University of Technology, Göteborg, Sweden, 1982.

[3] O. Axelsson, A survey of numerical methods for convection-diffusion equations, In Proceedings of XIV National Summer School on Numerical Solution Methods,Varna, 29.8-3.9, 1988.

[4] P. W. Hemker, G. I. Shishkin, and L. P. Shishkina, $\varepsilon$-uniform schemes with high-order timeaccuracy for parabolic singular perturbation problems, IMA J. Numer. Anal., 20(2000), no. 1, pp. 99-121. MR.1736952 (2000k:65139)

[5] H. Roos, M. Stynes, L. Tobiska, Numerical Methods for Singularly Perturbed Differential Equations, Springer, Heidelberg, 1996. MR1477665 (99a:65134)

[6] P. W. Hemker, A numerical study of stiff two-point boundary problems, Ph.D. thesis, Mathematical Center, Amsterdam, 1977. MR0488784 (58:8294)

[7] J. E. Marsden and M. J. Hoffman, Basic Complex Analysis, Second ed., W.H. Freeman, New York, 1987. MR913736 (88m:30001)

[8] M. Abramowitz and I. A. Stegun, eds., Handbook of Mathematical Functions. Applied Mathematics Series 55, National Bureau of Standards, Washington DC, 1964 . MR0167642 $(29: 4914)$

[9] M. V. Fedorjuk, The Method of Steepest Descent, Nauka, Moscow, 1977. MR0507923 $(58: 22580)$ 
[10] R.S.Varga, Matrix Iterative Analysis, Englewood Cliffs, NJ, Prentice Hall, 1962. MR0158502 (28:1725)

[11] C. Johnson, U. Nävert, and J. Pitkäranta, Finite element methods for linear hyperbolic problems, Comp. Meth. Appl. Mech. Eng., 45 (1984), pp. 285-312. MR759811 (86a:65103)

Faculty of Natural Sciences, Mathematics and Informatics, The University of Nijmegen, Toernooiveld 1, NL 6525 ED Nijmegen, The Netherlands

E-mail address: axelsson@sci.kun.nl

Current address: Department of Informatics Technology, Uppsala University, Box 337, 75105

Uppsala, Sweden

E-mail address: Owe.Axelsson@it.uu.se

Department of Applied Mathematics, Kuban State University, P.O. Box 4102, KRASNODAR, 350080, RUSSIA

E-mail address: evg@math.kubsu.ru

Department of Applied Mathematics, Kuban State University, P.O. Box 4102, Krasnodar, 350080, Russia 